\title{
Proton NMR Study of Copolymer Composition in Styrene-Methyl Methacrylate System under Magnetic Saturation-Free Condition
}

\author{
Riichirô Chûjô, ${ }^{*}$ Hiroshi MAtsuo, and Yoshio INOUE \\ Department of Polymer Chemistry, Tokyo Institute of Technology, \\ 12-1, Ookayama 2-chome, Meguro-ku, Tokyo 152, Japan.
}

(Received April 14, 1977)

\begin{abstract}
In the ${ }^{1} \mathrm{H}$ NMR spectroscopy of styrene-methyl methacrylate copolymers, relative intensity values are influenced by the effect of magnetic saturation if the effective $\mathrm{H}_{1}$ field is larger than $50 \mathrm{db}$. However, the spectra in the saturation-free condition are poor in signal-to-noise ratios. Multiscanned spectra were observed in the saturation-free condition, and the numerical data of the relative intensities from multiscanned spectra show that the copolymerization process obeys a simple Markovian process in the selection of monomer species, while it does a Bernoullian trial in the tactic placement.
\end{abstract}

KEY WORDS Copolymer / Styrene / Methyl Methacrylate / Proton NMR / Magnetic Saturation / Markovian Process / Bernoullian Trial /

Styrene-methyl methacrylate copolymers have been very widely studied by ${ }^{1} \mathrm{H}$ NMR among binary copolymers in order to elucidate their copolymerization kinetics, microstructure, sequence distributions and so on. ${ }^{1-7}$ The methoxyl proton peaks have been used mainly as the key signals in the study for these objectives. These peaks are certainly suitable for use for many reasons such as simple features due to the lack of spin-spin coupling with other protons, large chemical shifts due to the ring current effect of the phenyl groups in adjacent monomer units. However, there are disadvantage in the use of these peaks, namely, the partial coalescence with the peaks from other aliphatic protons. In order to overcome this disadvantage, the integral intensities of the peaks have been estimated by subtraction of those of the phenyl peaks from those of all the alliphatic peaks (a more definite algorithm will be shown in the Results and Discussion). The present authors believe that disagreement of the results in the studies mentioned at beginning of this paper originates from this subtraction procedure, since magnetic saturation factors may be different from each other

\footnotetext{
* To whom correspondence should be addressed.
}

between the peaks owing to the phenyl and the aliphatic protons in the copolymer, and the integral intensities of these peaks may not be proportional to corresponding mole numbers. Thus, a lack of understanding of these factors may lead to incorrect conclusions.

In this paper, the saturation-free condition of copolymers will be studied first. Following that, integral intensities will be measured under the suturation-free condition. Finally, the copolymerization kinetics of the systems will be discussed according to the data of the integral intensities.

\section{EXPERIMENTAL}

Styrene and methyl methacrylate monomers were mixed well after removal of the polymerization inhibitor. The mixed monomers were introduced into a tube containing azobisisobutylnitrile. Degassing and nitrogen blowing processes were repeated three times. After sealing the tube, the samples were polymerized at $60^{\circ} \mathrm{C}$ for three hours. The polymerized samples were diluted with benzene and poured into petroleum ether. The precipitates were filtered, dried in vacuo, dissolved in benzene, and freeze-dried. 
Table I. Copolymerization data of the samples used in this work

\begin{tabular}{cccc}
\hline $\begin{array}{c}\text { Sample } \\
\text { no. }\end{array}$ & $\begin{array}{c}\text { Molar feed } \\
\text { ratio, S/M }\end{array}$ & $\begin{array}{c}\text { Polymerization } \\
\text { time, hr }\end{array}$ & $\begin{array}{c}\text { Conversion, } \\
\%\end{array}$ \\
\hline 1 & 1.948 & 1.9 & 6.35 \\
2 & 1.551 & 1.5 & 6.09 \\
3 & 0.927 & 1.2 & 4.59 \\
4 & 0.631 & 1.0 & 4.37 \\
5 & 0.459 & 1.0 & 4.13 \\
\hline
\end{tabular}

Six kinds of copolymers having different monomer feed ratios were prepared. The copolymerization data are summarized in Table I for five of these six samples. The remaining sample was used for a preliminary test of the dependence of the relative intensities of NMR peaks on the magnitude of the effective magnetic field (feed ratio of this sample is 1.24).

The ${ }^{1} \mathbf{H}$ NMR spectra of the copolymers were measured in deuterated chloroform $(15 \%(\mathrm{w} / \mathrm{v}))$ with a JNM-PS-100 spectrometer. Sweep rates were fixed at $4.32 \mathrm{~Hz} / \mathrm{sec}$. The spectra with a single scan were measured by the external lock method. The aldehydic proton peaks of $m$ phthalic-di-aldehyde, which is added to the system, was used as a lock signal in the measurement of multiscanned spectra. A JEOL-EC-6 computer was linked to the spectrometer for the purpose of accumulation.

\section{RESULTS AND DISCUSSION}

In Figure 1 are shown typical ${ }^{1} \mathrm{H}$ NMR spectra of styrene-methyl methacrylate copolymers. The three peaks designated by $X, Y$, and $Z$ are those for the methoxyl protons. (These symbols are identical with those used in the work of Ito and Yamashita. ${ }^{4}$ In the paper of Harwood and Ritchey $^{3} \mathbf{A}, \mathbf{B}$, and $\mathrm{C}$ were used instead of $\mathrm{X}$, $Y$, and $Z$, respectively). The peak $Z$ partly coalesces with the peaks located at the higher side of the field. For evaluating the relative intensity of the peak $Z$, we must therefore use the method adopted by many authors; ${ }^{3,4}$ the relative (normalized) intensities $f_{\mathrm{X}}, f_{\mathrm{Y}}$, and $f_{\mathrm{Z}}$ of the peaks $\mathrm{X}, \mathrm{Y}$, and $\mathrm{Z}$ are calculated by

$$
\begin{aligned}
& f_{\mathrm{X}}=40 I_{\mathrm{X}} / 3\left(5 I_{\mathrm{A} 1 \mathrm{i}}-3 I_{\mathrm{Ph}}\right) \\
& f_{\mathrm{Y}}=40 I_{\mathrm{Y}} / 3\left(5 I_{\mathrm{Ali}}-3 I_{\mathrm{Ph}}\right) \\
& f_{\mathrm{Z}}=1-f_{\mathrm{X}}-f_{\mathrm{Y}}
\end{aligned}
$$

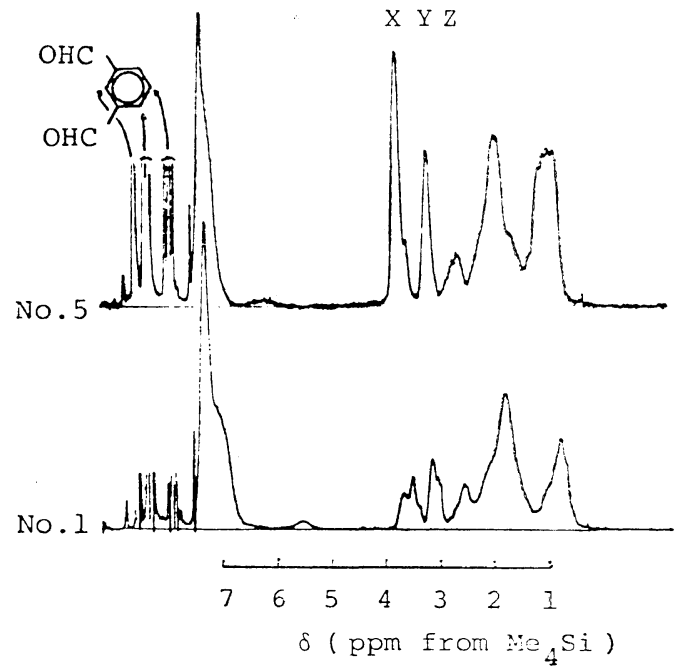

Figure 1. ${ }^{1} \mathrm{H}-\mathrm{NMR}$ spectra of styrene-methyl methacrylate copolymers (Samples I (bottom) and $\mathrm{V}$ (top)) in $\mathrm{CDCl}_{3}$ at $70^{\circ} \mathrm{C}$. These traces are the results of the accumulation of 105 (Sample I) and 64 (Sample V) times.

with the aid of the observed values of integral intensities $I_{\mathrm{X}}, I_{\mathrm{Y}}, I_{\mathrm{Ali}}$, and $I_{\mathrm{Ph}}$ of the peaks X, $\mathrm{Y}$ all of the aliphatic peaks (including $\mathrm{X}, \mathrm{Y}$, and $\mathrm{Z}$ ), and the phenyl peak, respectively. More precisely, $I_{\mathrm{Ph}}$ is the integral intensity of the peak located at $\delta$ about $3 \mathrm{ppm}$, which is assigned to the phenyl protons in the styrene units of the copolymers. $I_{\mathrm{A} 1 \mathrm{i}}$ is the sum of the integral intensities of all peaks of the copolymers except for the peak of the phenyl protons. Equation 1 is, of course, based on the proportionality between the integral intensities of individual peaks and the mole numbers of corresponding chemical species. In order to test whether this linearity really holds, NMR measurements for one sample were carried out under various magnitudes of

$$
H_{\mathrm{eff}}=H_{1}+H_{\mathrm{m}} \text {. }
$$

In eq $2 H_{1}$ and $H_{\mathrm{m}}$ denote the intensity of the high frequency oscillating field and the low frequency modulation field, respectively. In Figure 2 are plotted experimentally obtained values of $f_{\mathrm{X}}, f_{\mathrm{Y}}, f_{\mathrm{Z}}$, and $n_{\mathrm{S}}$ against $H_{\mathrm{eff}}$. The quantity $n_{\mathrm{S}}$ is the composition ratio of styrene-methyl methacrylate. The constancy of these four quantities holds only in the region where $H_{\mathrm{eff}}$ is less than $50 \mathrm{db}$. Some of the points $\ominus, \Theta, \bigcirc$, and (1) are the data obtained from a single scan. We find that the effect of the magnetic saturation 
Proton NMR of Styrene-Methyl Methacrylate Copolymer

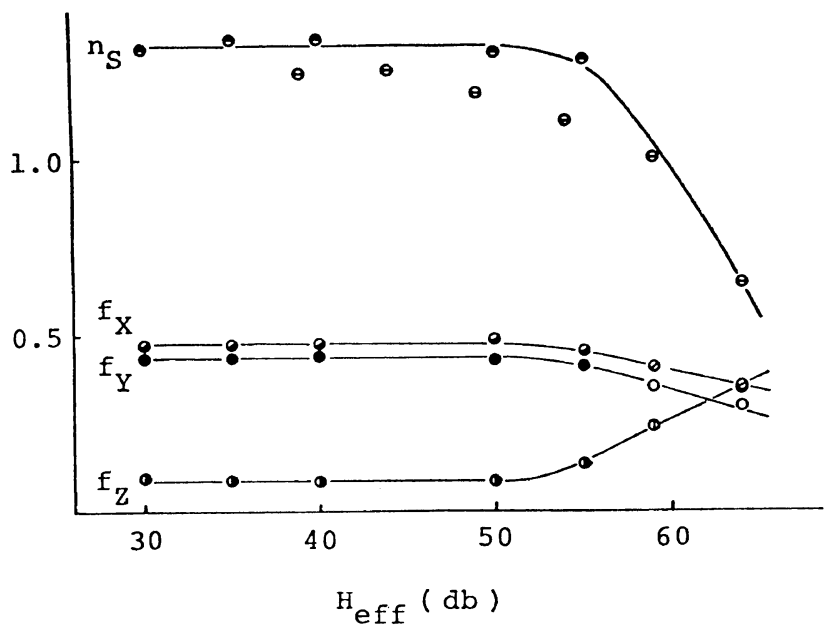

Figure 2. Dependence of the relative intensities $f_{\mathrm{X}}, f_{\mathrm{Y}}$, and $f_{\mathrm{Z}}$ of the methoxyl peaks and experimentally obtained composition ratio, $n_{\mathrm{S}}$ on the magnitude $H_{\text {eff }}$ of effective $H_{1}$ field. Initial copolymerization condition: a monomer feed ratio, 1.24. $(\Theta, \oslash, \bigcirc$, and (1), data from single scan; remainings, data from multiscan.)

Table II. Experimental condition and result of the NMR measurement

\begin{tabular}{ccccccc}
\hline \multirow{2}{*}{$\begin{array}{c}\text { Sample } \\
\text { no. }\end{array}$} & $\begin{array}{c}\text { Accumulation, } \\
\text { time }\end{array}$ & $\begin{array}{c}\text { Molar com- } \\
\text { position } \\
n_{\mathrm{S}}, \mathrm{S} / \mathrm{M}\end{array}$ & \multicolumn{3}{c}{ Integral intensities } & \multirow{2}{*}{$4 f_{\mathrm{x}} f_{\mathrm{z}} / f_{\mathrm{Y}^{2}}$} \\
\cline { 3 - 6 } & & 1.682 & 0.430 & 0.449 & 0.121 & 1.031 \\
1 & 105 & 1.440 & 0.456 & 0.443 & 0.101 & 0.935 \\
2 & 68 & 0.982 & 0.509 & 0.397 & 0.094 & 1.220 \\
3 & 75 & 0.760 & 0.558 & 0.373 & 0.068 & 1.111 \\
4 & 64 & 0.590 & 0.608 & 0.340 & 0.052 & 1.098 \\
5 & 64 & & & & &
\end{tabular}

gradually increases with an increasing of $H_{\text {eff }}$ in the larger $H_{\text {eff }}$ region.

In Figure 2 there are included the data obtained from the multiscan. Multiscanning was carried out in order to improve $\mathrm{S} / \mathrm{N}$ (signal-tonoise ratio) in the region with smaller values of $H_{\text {eff }}$. This is a general feature both in the low molecular weight compounds ${ }^{8}$ and in high polymers. ${ }^{9}$ As a result, NMR measurements are frequently carried out at larger values of $H_{\text {eff. }}$ Although we could not find any description of the value of $H_{\text {eff }}$ in already published works on styrene-methyl methacrylate copolymers, we inferred that an $H_{\text {eff }}$ larger than $50 \mathrm{db}$ had been applied there on making a comparison between the spectra shown in those works and the spectra obtained in the present work.

We may conclude that the NMR measurement must be carried out under the condition of
$H_{\text {eff }}<50 \mathrm{db}$ in a multiscan of styrene-methyl methacrylate copolymers, even in ${ }^{1} \mathrm{H}$ NMR. Thus the relative intensities, $f_{\mathrm{X}}, f_{\mathrm{Y}}$, and $f_{\mathrm{Z}}$ were determined under a suitable condition for each of the five samples. These conditions and results summarized in Table II.

According to the assignment proposed by Ito and Yamashita, ${ }^{4}$ the relative intensities are interrelated with probability parameters by

$$
\begin{aligned}
& f_{\mathrm{X}}=\left(1-\sigma p_{\mathrm{MS}}\right)^{2} \\
& f_{\mathrm{Y}}=2 \sigma p_{\mathrm{MS}}\left(1-\sigma p_{\mathrm{MS}}\right) \\
& f_{\mathrm{Z}}=\left(\sigma p_{\mathrm{MS}}\right)^{2}
\end{aligned}
$$

where $p_{\mathrm{MS}}$ is a conditional probability of an addition of $S$ (styrene) monomer on $M$ (methyl methacrylate) growing end, and $\sigma$ is a probability of coisotactic addition of $S$ on $M$. The constancy of these two parameters means that the 
selection of monomer species obeys a simple Markovian process while the tactic addition obeys the Bernoullian trial in copolymerization. Such a copolymerization mechanism seems reasonable. Nevertheless, already published numerical data ${ }^{4}$ do not satisfy the condition

$$
4 f_{\mathrm{X}} f_{\mathrm{Z}} / f_{\mathrm{Y}}{ }^{2}=1
$$

which is required from eq 3 . On the other hand, the numerical values in the column $4 f_{\mathrm{X}} f_{\mathrm{Z}} / f_{\mathrm{Y}}{ }^{2}$ in Table II are rather close to unity. This means that the above mentioned mechanism is justified from ${ }^{1} \mathrm{H}$ NMR spectra in a multiscan.

The numerical data of the last column in Table II distribute somewhat around unity. In order to discuss the polymerization mechanism more clearly, an estimation of the approximate joint $r_{\mathrm{M}} r_{\mathrm{S}}$ confidence interval is required. This procedure of estimation has been described in the work of Tidwell and Mortimer ${ }^{10}$ as an ap-

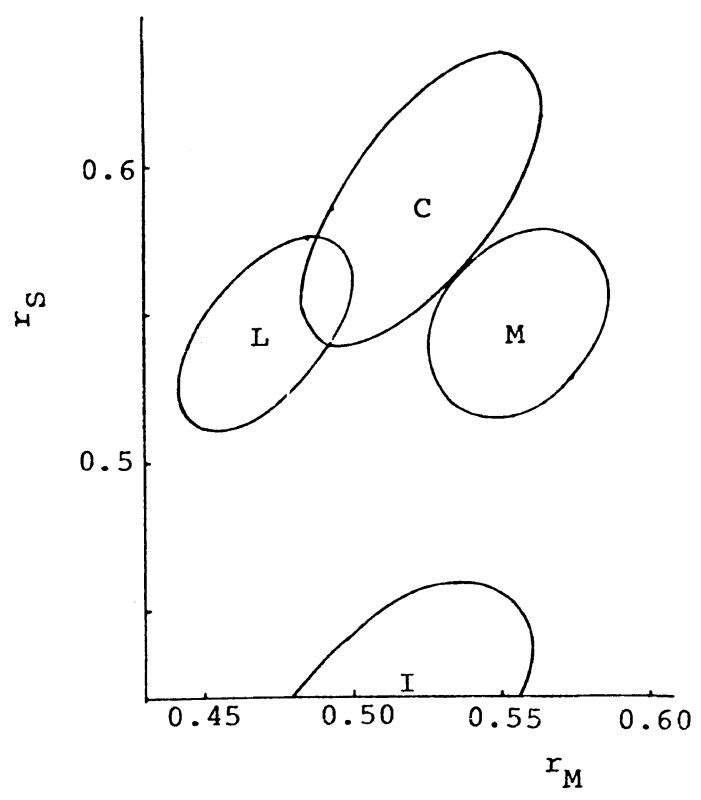

Figure 3. Approximate joint $r_{M} r_{S}$ confidence intervals calculated from (C) the data of present work, (L) the data of Mayo and Lewis, (M) the data of Meyer, and (I) the data of Ito and Yamashita. L, M, and I were reproduced from the work of Tidwell and Mortimer. plication of nonlinear least square analysis. The symbol $r_{\mathrm{S}}$ and $r_{\mathrm{M}}$ denote the monomer reactivity ratios of styrene and methyl methacrylate, respectively. The calculated results are shown in Figure 3 with symbol C. In Figure 3 are also reproduced the results calculated by Tidwell and Mortimer $^{11}$ for the data of combustion $(\mathrm{C}, \mathrm{H})$ analysis by Mayo and Lewis, ${ }^{12}$ for the data of neutron activation analysis by Meyer, ${ }^{13}$ and for the NMR data by Ito and Yamashita. ${ }^{4}$ These are represented by symbols L, M, and I, respectively. Comparing these data with each other, we can say that the data (C) from NMR measurement with low $H_{\mathrm{eff}}$ is closer to the data (L and $M$ ) obtained by other methods than NMR. We should like to emphasize again the importance of NMR measurements with lower magnitude of $H_{\text {eff }}$ and the reasonableness of the polymerization mechanism corresponding to eq 3 .

\section{REFERENCES}

1. F. A. Bovey, J. Polym. Sci., Part A-1, 4, 631 (1966).

2. Y. Kato, N. Ashikari, and A. Nishioka, Bull. Chem. Soc. Jpn., 37, 1630 (1964).

3. H. J. Harwood and W. M. Ritchey, J. Polym. Sci., Part B, 3, 419 (1965).

4. K. Ito and Y. Yamashita, J. Polym. Sci., Part $B, 3,625$ (1965).

5. V. D. Moche1, Macromolecules, 2, 537 (1969).

6. M. Hirooka, H. Yabuuchi, J. Iseki, and Y. Nakai, J. Polym. Sci., Part A-1, 6, 1381 (1968).

7. A. D. Jenkins and M. G. Rayner, Eur. Polym. $J .$, 8, 221 (1972).

8. K. Hatada and Y. Terawaki, J. Chem. Soc. Jpn. (Ind. Chem. Sect.), 71, 1163 (1968).

9. K. Hatada, K. Ota, Y. Terawaki, and H. Yuki, J. Chem. Soc. Jpn. (Ind. Chem. Sect.), 711168 (1968).

10. P. W. Tidwell and G. A. Mortimer, J. Polym. Sci., Part A, 3, 369 (1965).

11. P. W. Tidwell and G. A. Mortimer, J. Macromol. Sci., C4, 281 (1970).

12. F. R. Mayo and F. M. Lewis, J. Am. Chem. Soc., 66, 1594 (1944).

13. V. E. Meyer, J. Polym. Sci., Part A-1, 4, 2819 (1966). 